\title{
Partial Detection for Multiple Antenna Cooperation
}

\author{
Kiarash Amiri, Joseph R. Cavallaro \\ Center for Multimedia Communication \\ Department of Electrical and Computer Engineering \\ MS-366, Rice University, 6100 Main St., Houston, TX 77005 \\ \{kiaa, cavallar\}@rice.edu
}

\begin{abstract}
Multi-antenna relays can significantly increase the speed and reliability of wireless systems. However, because of the complexity of MIMO detection, there is considerable overhead in implementing a MIMO relay if the conventional detect-and-forward strategy is used. To address this challenge, we propose a novel cooperative partial detection (CPD) strategy that partitions the detection task between the relay and the destination. CPD leverages the structure of the tree-based close-toML MIMO detectors, and modifies the tree traversal so that instead of visiting all the levels of the tree, only a subset of the levels, thus a subset of the transmitted streams, are visited. This novel approach reduces the tree levels, i.e. dimensions, in both the relay and the destination. Moreover, CPD provides a flexible method to control the level of partitioning between the relay and the destination, and thus, adjust the detection computational complexity in the relay and the destination. Monte-Carlo simulation results show that, under equal transmit power and complexity constraint in the destination, CPD achieves a better BER performance compared to the non-relay scenario, with limited computational overhead in the relay.
\end{abstract}

\section{INTRODUCTION}

Cooperative communications, and in particular relay channels, reemerged as a form of diversity in uplink scenarios [1]. Following this attempt, different relaying protocols, such as decode-and-forward and amplify-and-forward, were studied and compared for different cooperation scenarios [2]. Also, with the promising results of MIMO point-to-point communications [3], MIMO systems have been playing a significant role in a wide variety of wireless standards, and thus, various detection algorithms, mostly based upon sphere detection and tree-based searches, have been proposed to reduce the complexity of detection in MIMO systems [4], [5], [6].

More recently, there have been some attempts to study the theoretical benefits and bounds on deploying MIMO nodes in cooperative scenarios, both as relays and as source/destination pairs. In doing so, lower bounds and upper bounds for MIMO relay networks were given in [7], [8], and capacity scaling factors were derived for multi-hop MIMO relays [9].

Full detect-and-forward or decode-and-forward in the relay can require a significant amount of resources in MIMO cooperative communications, particularly if the relay chooses to perform a closeto-optimum detection. This effect becomes more important for the cases where the relay is a mobile MIMO user, rather than a fixed dedicated relay, giving up some of its resources to assist another MIMO user. Therefore, it is crucial to distribute the detection task between the relay and the destination in such a way that the relay does not need to spend too much of its processing and transmit power, and yet, can enhance the performance compared to a non-relay scenario.

In order to address this challenge, we propose a novel cooperative partial detection scheme in MIMO relay channels, which leverages the inherent tree-based structure of close-to-optimum MIMO detection schemes. In our proposed method, the relay node, instead of applying the conventional full detection, performs a partial detection by splitting the detection tree into two portions, and detecting only one portion of the tree. Then, the relay forwards the detected streams of the message to the destination. Finally, the destination utilizes the multiple available copies and generates new detection trees to detect the transmitted message. We will show that this cooperative detection scheme can achieve better performance than the direct-link non-relay scenario, with limited computation overhead in the relay and without any extra computation power in the destination.

The proposed detection strategies utilize the inherent structure of the close-to-optimum MIMO detection schemes by splitting them into smaller dimensions, and thus, lowering the complexity. We propose a partial $K$-best detection scheme, which is designed based on the practical limitations of wireless devices. This detection scheme, which has a lower dimension than that of the full detector, is used in the relay for partial detection, and can result in considerable complexity reduction. Furthermore, instead of performing a full largedimension detection, the destination node performs the detection using two smaller dimension detection trees. This method, overall, leads to reducing the total complexity.

This paper is organized as follows: Section II covers the system model definition, an overview of close-to-ML $K$-best MIMO detection schemes is given in section III, and the proposed partial $K$-best technique is described in section IV. The cooperative partial detection algorithm is presented in section $\mathrm{V}$, and the computational complexity of this technique is studied in section VI. Monte-Carlo simulation results of this scheme are presented in section VII, and finally, the paper concludes with section VIII.

\section{SYSTEM MODEL}

We assume a three node network: the source, relay and destination, denoted by S, R and D; respectively. We further assume that the source, relay and destination are equipped with $M_{s}, M_{r}$ and $M_{d}$ antennas; respectively. Given the practical limitations of deploying full duplex radios, we assume the relay operates in half-duplex mode. The communication between the source and the destination is performed in two time slots. In the first time slot, the source broadcasts its message to both the relay and the destination; and in the second time slot, the relay, using a $M_{r u} \leq M_{r}$ subset of its antennas, transmits its message to the destination while the source is silent.

The received signals at the relay and destination at the end of the first time slot are given by

$$
\begin{aligned}
\mathbf{y}_{r} & =\sqrt{\frac{\mathrm{SNR}_{s r}}{M_{s}}} \mathbf{H}_{s r} \mathbf{x}_{s}+\mathbf{n}_{r}, \\
\mathbf{y}_{d}^{(1)} & =\sqrt{\frac{\mathrm{SNR}_{s d}}{M_{s}}} \mathbf{H}_{s d} \mathbf{x}_{s}+\mathbf{n}_{d}^{(1)} .
\end{aligned}
$$

Likewise, the received signal at the destination at the end of the second time slot is given by

$$
\mathbf{y}_{d}^{(2)}=\sqrt{\frac{\mathrm{SNR}_{r d}}{M_{r u}}} \mathbf{H}_{r d} \mathbf{x}_{r}+\mathbf{n}_{d}^{(2)}
$$


where superscripts ${ }^{(1)}$ and ${ }^{(2)}$ are used to distinguish the first and second time slots. Since the relay receives only at the end of the first time slot, no superscript is used for the relay. In Eq. (1) to (3), $M_{r u}$ corresponds to the number of utilized antennas in the relay during the second time slot; hence $M_{r u} \leq M_{r}$. The noise vectors, $\mathbf{n}_{r}, \mathbf{n}_{d}^{(1)}$ and $\mathbf{n}_{d}^{(2)}$ are of size $M_{r}, M_{d}$ and $M_{d}$, with each of their elements chosen from a complex symmetric Gaussian variable $\mathcal{C} N(0,1)$. We also assume that each element of the $\mathbf{x}_{s}, \mathbf{x}_{r}$ and $\mathbf{x}_{d}$ vectors are chosen from a QAM modulation, $\Omega$, with the modulation set size of $w=|\Omega|$, and average power constraint of $E\left[x_{i}{ }^{2}\right]=1$.

As illustrated in Figure 1, the $\mathbf{H}_{s r}, \mathbf{H}_{r d}$ and $\mathbf{H}_{s d}$ are matrices of sizes $M_{r} \times M_{s}, M_{d} \times M_{r u}$ and $M_{d} \times M_{s}$; and correspond to the channel matrices between the source and the relay, relay and the destination, and source and the destination, respectively. All these channel matrices have independent elements, each drawn from a circularly symmetric Gaussian random distribution with zero mean and variance of one, $\mathcal{C} N(0,1)$. We make the practically feasible assumption that the $\mathbf{H}_{s r}$ matrix is known at the relay; and $\mathbf{H}_{s d}$ and $\mathbf{H}_{r d}$ matrices are known at the destination node; thus, only the receivers of each communication link have complete channel knowledge.

The signal-to-noise ratios, SNR, at each of the received antennas of the relay and destination are defined as

$$
\mathrm{SNR}_{s r}=\frac{\mu P}{\left(d_{s r}\right)^{\alpha}}, \mathrm{SNR}_{s d}=\frac{\mu P}{\left(d_{s d}\right)^{\alpha}}, \mathrm{SNR}_{r d}=\frac{(1-\mu) P}{\left(d_{r d}\right)^{\alpha}},
$$

where $\alpha$ is the path loss exponent, and usually changes between 2 and 6 . The above SNR equations imply that the sum transmit power from the source and the relay is set to $P$, and is split with a proportion factor of $0<\mu \leq 1$, such that the source uses $\mu P$ and the relay uses $(1-\mu) P$.

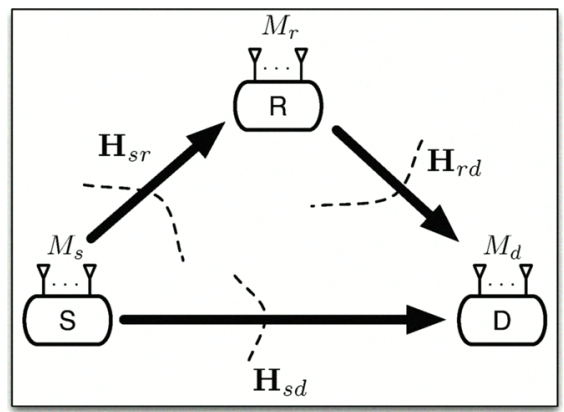

Fig. 1. A relay network with three nodes: source, relay and destination. The respective channel matrices are denoted by $\mathbf{H}_{s r}, \mathbf{H}_{r d}$ and $\mathbf{H}_{s d}$.

\section{DETECTION IN MIMO SYSTEMS}

The maximum-likelihood (ML) detector for spatially multiplexed MIMO systems imposes an exponential computational increase on the receiver; thus, the sphere detector technique has been proposed to dramatically reduce the complexity of MIMO detectors [10]. A suboptimal architecture-friendly variation of sphere detector, known as the $K$-best detector, was later proposed in [4], [5]. Unlike the sphere detector, which offers a fixed average computational complexity per SNR, the $K$-best detector's complexity depends on the value of $K$, where higher $K$, i.e. more computation, closes the gap with ML; therefore, it provides a measure to control the "quality" and "accuracy" of the detector.
The $K$-best detector offers a considerably higher performance compared to linear detectors, e.g. zero-forcing and MMSE. It also lends itself to architecture-friendly implementations that are amenable to parallelization, and offers fixed latency; while its BER performance can become arbitrarily close to ML performance by choosing the right value of $K$ for the specific SNR regime. Given the advantages of the $K$-best detector, we choose $K$-best to perform MIMO detection throughout this paper.

Assuming $\tilde{\mathbf{f}}=\tilde{\mathbf{A}} \tilde{\mathbf{b}}+\tilde{\mathbf{g}}$, as a generalization of Eq. (1-3), the $K$ best technique can be used to solve the following integer least-squares problem

$$
\min _{\tilde{\mathbf{b}} \in \Omega^{m}}\|\tilde{\mathbf{f}}-\tilde{\mathbf{A}} \tilde{\mathbf{b}}\|_{2}^{2} .
$$

Prior to performing the $K$-best detection, the channel matrix and the received vector are decomposed into a real-valued matrix and vector:

$$
\left(\begin{array}{c}
\Re(\tilde{\mathbf{f}}) \\
\Im(\tilde{\mathbf{f}})
\end{array}\right)=\left(\begin{array}{cc}
\Re(\tilde{\mathbf{A}}) & -\Im(\tilde{\mathbf{A}}) \\
\Im(\tilde{\mathbf{A}}) & \Re(\tilde{\mathbf{A}})
\end{array}\right)\left(\begin{array}{c}
\Re(\tilde{\mathbf{b}}) \\
\Im(\tilde{\mathbf{b}})
\end{array}\right)+\left(\begin{array}{c}
\Re(\tilde{\mathbf{g}}) \\
\Im(\tilde{\mathbf{g}})
\end{array}\right),
$$

where $\Re($.$) and \Im($.$) denote the real and imaginary parts of (.). Eq.$ (6) can be represented by

$$
\mathbf{f}=\mathbf{A b}+\mathbf{g}
$$

The norm in (5) can be re-written as [10]

$$
\begin{aligned}
D(\mathbf{b}) & =\|\mathbf{f}-\mathbf{A} \mathbf{b}\|^{2} \\
& =\left\|\mathbf{Q}^{H} \mathbf{f}-\mathbf{R} \mathbf{b}\right\|^{2}=\sum_{i=2 m}^{1}\left|f_{i}{ }^{\prime}-\sum_{j=i}^{2 m} R_{i, j} b_{j}\right|^{2},
\end{aligned}
$$

where $\mathbf{A}=\mathbf{Q R}, \mathbf{Q} \mathbf{Q}^{H}=\mathbf{I}$ and $\mathbf{f}^{\prime}=\mathbf{Q}^{H} \mathbf{f}$. Note that the transition in (8) is possible through the fact that $\mathbf{R}$ is an upper triangular matrix. The real-valued decomposition, channel ordering, $\mathrm{QR}$ decomposition, and computation of $\mathbf{f}^{\prime}=\mathbf{Q}^{H} \mathbf{f}$ are conventionally referred to as preprocessing operations, most of which happen at the channel updating rate rather than the data processing rate. As shown in the earlier work [4], [5], the norm in (8) can be computed in a step-by-step tree search, where at each level, the best $K$ candidates are kept and tree traversal continues until all the tree levels, i.e. transmit antennas, are visited. Moreover, throughout this paper, we assume that all the $\ell^{2}$ norms in (8) are approximated with the $\ell^{1}$ norms since it leads to simplifying the detector architecture, with very negligible performance loss [11].

For systems with channel coding, the transmitter consists of an outer error correction encoder, e.g. Turbo or LDPC encoder, followed by a constellation mapper which maps the encoded bits to complex modulation symbols, e.g. 16-QAM or 64-QAM. The decoder in the receiver requires soft information per transmitted bits. The soft information, typically Log-likelihood Ratio (LLR), passed from the detection block to the decoding block is obtained by

$$
L_{E}\left(u_{k} \mid \tilde{\mathbf{f}}\right)=\ln \frac{P\left[u_{k}=+1 \mid \tilde{\mathbf{f}}\right]}{P\left[u_{k}=-1 \mid \tilde{\mathbf{f}}\right]}
$$

where $k=1, \ldots, m \log w$.

Soft information can be generated using a list of possible vector candidates $\mathcal{L}$, which in our case is the set of the surviving candidates of the last level of the detection tree. Once this list is generated, LLR values of Eq. (9) are computed and passed to the decoder [12]:

$$
\begin{gathered}
L_{E}\left(u_{k} \mid \tilde{\mathbf{f}}\right) \quad \approx \frac{1}{2} \max _{\mathbf{u} \in \mathcal{L} \cap \mathbf{U}_{\mathbf{k},+1}}\left\{-\frac{1}{\sigma^{2}}\|\tilde{\mathbf{f}}-\tilde{\mathbf{A}} \cdot \tilde{\mathbf{b}}\|^{2}\right\} \\
-\frac{1}{2} \max _{\mathbf{u} \in \mathcal{L} \cap \mathbf{U}_{\mathbf{k},-1}}\left\{-\frac{1}{\sigma^{2}}\|\tilde{\mathbf{f}}-\tilde{\mathbf{A}} \cdot \tilde{\mathbf{b}}\|^{2}\right\},
\end{gathered}
$$


where $\mathcal{L}$ is the list of possible vectors, $\sigma^{2}$ is the noise variance, $\mathbf{U}_{\mathbf{k},+1}$ is the set of $2^{m \log w-1}$ bits of vector $\mathbf{u}$ with $u_{k}=+1$, while $\mathbf{U}_{\mathbf{k},-1}$ is similarly defined. In order to facilitate the cooperative detection strategy, without imposing large computational overhead onto the relay, we propose partial $K$-best detector in the next section.

\section{Partial $K$-best Detection}

Our proposed partial $K$-best detector requires similar preprocessing operations as that of the conventional $K$-best detector. However, rather than doing the decomposition in (6), where the in-phase and quadrature components are separated in two groups; we use Modified Real-valued Decomposition (M-RVD) [13], so that each in-phase component is followed by its quadrature component, $\mathbf{b}=\left[\Re\left(\tilde{b}_{1}\right), \Im\left(\tilde{b}_{1}\right), \Re\left(\tilde{b}_{2}\right), \Im\left(\tilde{b}_{2}\right), \ldots, \Re\left(\tilde{b}_{m}\right), \Im\left(\tilde{b}_{m}\right)\right]^{T}$. Note that using this ordering, as we see later in this section, helps in separating different antennas. The corresponding channel matrix columns need to be swapped accordingly as shown in [13].

Next, the QR decomposition triangularizes the equivalent realvalued channel matrix, and the tree traversal starts from the top level, $i=2 m$, where $m$ is the number of transmit antennas, and thus, $2 m$ is the number of columns of the new real-valued channel matrix. Unlike the conventional $K$-best method, the tree traversal of the partial $K$-best method terminates in one of the middle levels, and the corresponding minimum distance at that level is considered as the "partial" detected symbol vector. We call the number of visited antennas the expansion factor, ef. Figure 2 shows this process for an example case with 16-QAM modulation, and expansion factor of 2. Due to the real-valued decomposition, each transmit antenna corresponds to two tree levels. Note that if we did not choose to use M-RVD, the four consecutive levels of Figure 2 would have represented the quadrature components of the four antennas; whereas, with M-RVD, these four levels correspond to both the in-phase and quadrature components of two of the antennas.

\section{Cooperative Partial Detection (CPD) With MiMo RELAYS}

One of the prominent methods of using relays has been full detect (and/or decode)-and-forward, FDF, which would impose a significant overhead onto the relay in the case of (virtual) MIMO scenarios. In order to address this challenge, we propose a novel cooperative partial detection, CPD, scheme in MIMO systems. We first consider an uncoded system; then, we extend it to a system with channel coding.

\section{A. Uncoded Detection}

We will assume two transmission time slots: during the first time slot, the source transmits its vector message, $\mathbf{x}_{s}$ in Eq. (1) and (2), to the relay and destination. The relay receives $\mathbf{y}_{r}$ of Eq. (1), and partially detects the message through the process described in section IV, i.e. it detects an $e f$-length subset of the elements of the transmit vector. In the second time slot, the relay forwards its detected vector of the first time slot, the $\mathbf{x}_{r}$, to the destination. The detection procedure in the destination is comprised of four steps, as shown in Figure 3:

1. The destination, using the copy that it received from the relay in the second time slot, $\mathbf{y}_{d}^{(2)}$, detects the $\mathbf{x}_{r}$ vector, and calls this detected vector, $\mathbf{x}_{d}^{(2)}$.

2. The $\mathbf{x}_{d}^{(2)}$ vector is cancelled from the $\mathbf{y}_{d}^{(1)}$ :

$$
\mathbf{y}_{d}^{(t m p)}=\mathbf{y}_{d}^{(1)}-\mathbf{H}_{s d}^{(1: e f)} \mathbf{x}_{d}^{(2)}
$$

3. Using $\mathbf{y}_{d}^{(t m p)}$, the remaining streams, i.e. those not detected in the relay and thus, not forwarded to the destination, are detected and called $\mathbf{x}_{d}^{(1)}$.

4. The two vectors, $\mathbf{x}_{d}^{(1)}$ and $\mathbf{x}_{d}^{(2)}$ are concatenated to form the final detected vector,

$$
\mathbf{x}_{d}=\left[\mathbf{x}_{d}^{(2)}, \mathbf{x}_{d}^{(1)}\right]
$$

Note that the detection in the first and third steps, in the destination, are performed using the typical $K$-best detector; whereas, the relay performs the partial $K$-best method of section IV. Therefore, there are three major detection parameters which may be lumped together in the $\mathbf{k}_{C P D}=\left(K_{r}, K_{d r}, K_{d s}\right)$ triple, where $K_{r}$ is the $K$ value for the partial $K$-best detection in the relay, and $\left(K_{d r}, K_{d s}\right)$ are the $K$ values for the $K$-best detection in the destination. $K_{d r}$ is the $K$ value for the $K$-best detection of $\mathbf{x}_{r}$ in step 1 , and $K_{s r}$ is the $K$ value used for the detection of the remaining streams in step 3.

\section{B. Coded Detection}

In order to extend the partial cooperative detection to coded systems, we employ the QRD-M technique [14], [15] in the destination to compute the soft information required in the decoder. Therefore, while the relay performs the regular partial detection described in section V-A, the destination performs a QRD-M detection in both of its internal detection steps. Therefore, the modified detection procedure, from section $\mathrm{V}-\mathrm{A}$, in the destination is:

1. The destination, using the copy that it received from the relay in the second time slot, $\mathbf{y}_{d}^{(2)}$, detects the $\mathbf{x}_{r}$ vector, and calls this detected vector, $\mathbf{x}_{d}^{(2)}$. Moreover, using the list of the surviving candidates of the last level of the tree, $\mathcal{L}^{(2)}$, compute the corresponding LLR values $\mathbf{L}_{E}^{(2)}$.

2. The $\mathbf{x}_{d}^{(2)}$ vector is cancelled from the $\mathbf{y}_{d}^{(1)}$ :

$$
\mathbf{y}_{d}^{(t m p)}=\mathbf{y}_{d}^{(1)}-\mathbf{H}_{s d} \mathbf{x}_{d}^{(2)}
$$

3. Using $\mathbf{y}_{d}^{(t m p)}$, the remaining streams, i.e. those not detected in the relay and thus, not forwarded to the destination, are detected and called $\mathbf{x}_{d}^{(1)}$. Then, using the list of the surviving candidates of the last level of the tree, $\mathcal{L}^{(1)}$, compute the corresponding LLR values $\mathbf{L}_{E}^{(1)}$.

4. Based on the power reordering of the streams that have been performed in the relay, the two vectors, $\mathbf{x}_{d}^{(1)}$ and $\mathbf{x}_{d}^{(2)}$ are concatenated to form the final detected vector,

$$
\begin{aligned}
\mathbf{x}_{d} & =\left[\mathbf{x}_{d}^{(2)}, \mathbf{x}_{d}^{(1)}\right] \\
\mathbf{L}_{E} & =\left[\mathbf{L}_{E}^{(2)}, \mathbf{L}_{E}^{(1)}\right] .
\end{aligned}
$$

\section{COMPLEXITY ANALYSIS}

In order to compare the complexity, we count the number of computations in the relay and destination. As discussed in [13], [16], the number of additions and compare-select operations to perform $K$-best is given by:

$$
\begin{aligned}
& C_{a d d}=2 w^{\prime}+2 w+2 K w^{\prime}(2 m-2)+K(m(2 m-1)-1), \\
& C_{\text {compare-select }}=f_{K}\left(K w^{\prime}\right)(2 m-3)+f_{K}(w)+f_{1}\left(K w^{\prime}\right),
\end{aligned}
$$

where $w^{\prime}=\sqrt{w}$, and $m$ is the number of transmit antennas, i.e. the transmit vector length. Also, we define $f_{K}(l)=K \times(l-K)$ where $f_{K}(l)$ is the number of compare-select operations required for finding the best $K$ candidates in a list of size $l$ if bubble sort is used. It was shown in [11] that bubble sort is a suitable VLSI implementation choice for a wide range of $K$-best detectors as long 


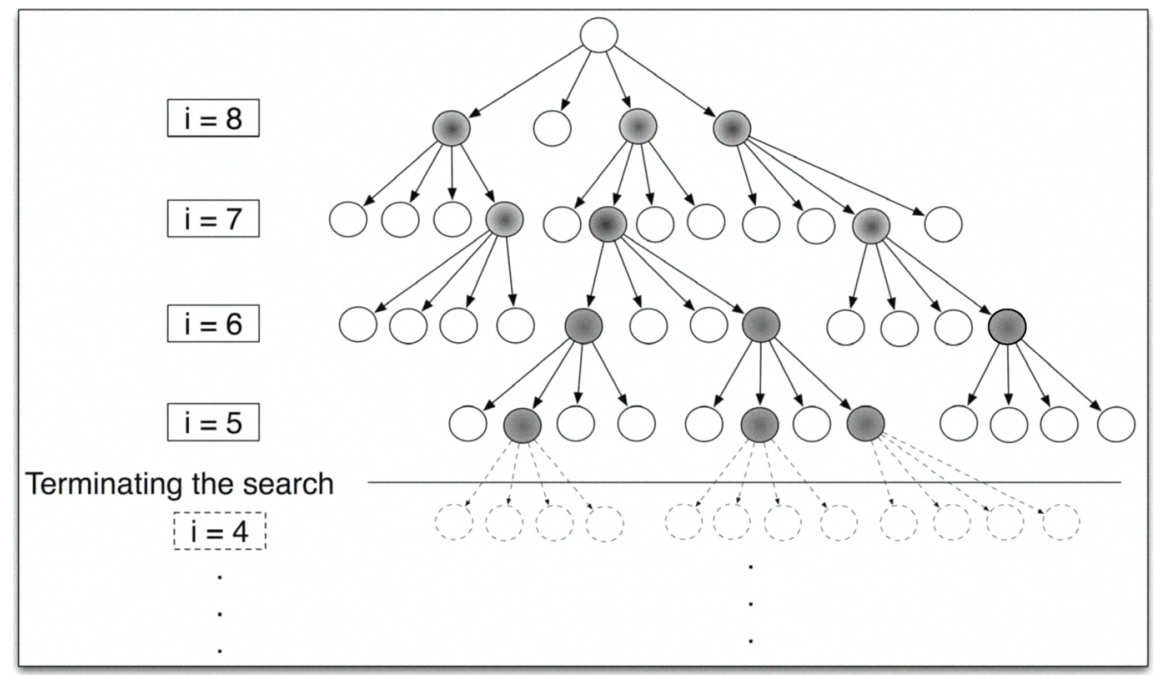

Fig. 2. The tree structure for a partial $K$-best MIMO detector with $K=3$ and expansion factor of two, ef $=2$. Real-valued decomposition (RVD) has been utilized, therefore, each node has $\sqrt{16}=4$ children for the example case of 16 -QAM modulation.
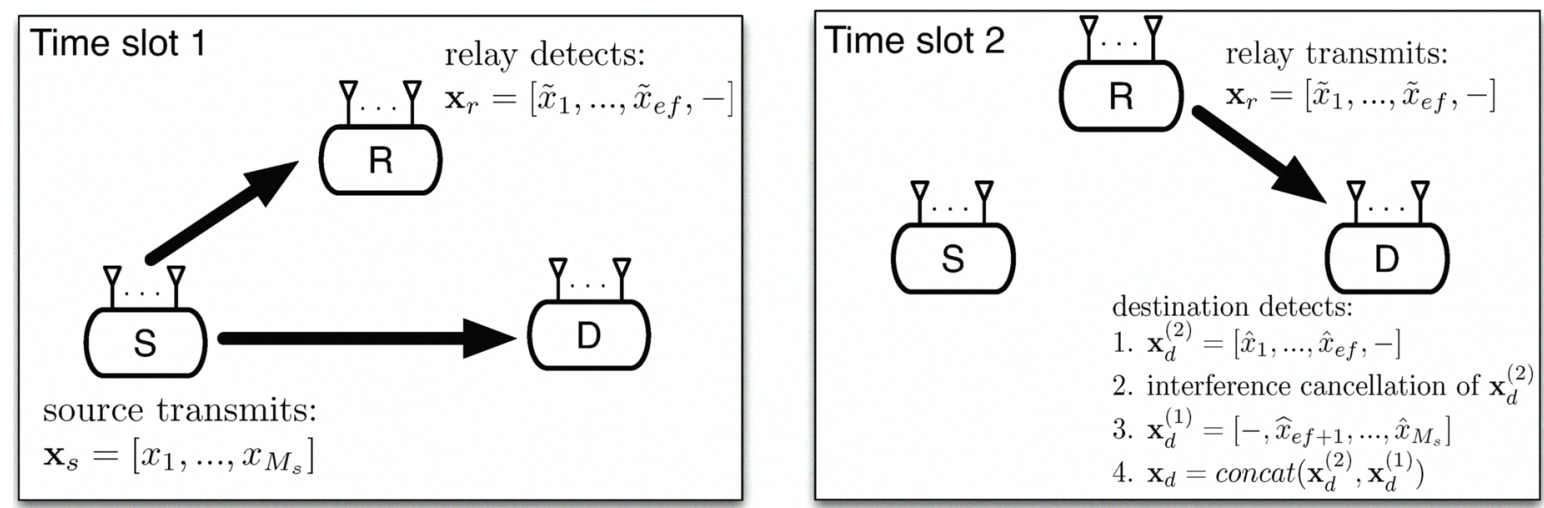

Fig. 3. Cooperative detection through MIMO relay node. In the first time slot, the relay receives a copy of the source multi-stream data, and partially detects it, and forwards the detected data. In the second time slot, the receiver first detects the copy received from the relay, then performs interference cancellation of the detected vector from the copy of the first time slot, and detects the remaining streams.

as $K$ is larger than $w^{\prime}$. Note that (16) and (17) do not account for the ordering and $\mathrm{QR}$ decomposition as those operations take place at a much slower rate compared to the core $K$-best detector unit. Also, the multiplications are replaced with shift-add operations as one term of the products are scaled integers of the modulation set.

Knowing the number of computations for addition and compareselect operations from Eq. (16) and (17), we can write the overall number of computations for one $K$-best detection as a function of $m, K, w$ :

$$
C_{K-b e s t}(m, w, K)=\theta \cdot C_{a d d}+\beta \cdot C_{\text {compare-select }},
$$

where we use $\theta$ and $\beta$ to represent the hardware-oriented costs for one real-valued adder and one real-valued compare-select operation, respectively.

Having derived the overall computation cost of the generic $K$ best detector of Eq. (5), we can compute the computation cost of the cooperative detection scheme of section $\mathrm{V}$. Therefore, given the parameters of the cooperative system, defined in section II, the numbers of operations required to perform the proposed cooperative partial detection, in the relay and destination, are

$$
\begin{aligned}
C_{C P D}^{R}\left(M_{s}, w, \mathbf{k}_{C P D}\right) & =C_{K-\text { best }}\left(e f, K_{r}, w\right), \\
C_{C P D}^{D}\left(M_{s}, w, \mathbf{k}_{C P D}\right) & =C_{K-\text { best }}\left(e f, K_{d r}, w\right) \\
& +C_{K-b e s t}\left(M_{s}-e f, K_{d s}, w\right) \\
& +C_{L L R}\left(M_{s}, K_{d}^{\prime}, w\right) \\
& +C_{L L R}\left(M_{s}-e f, K_{d}^{\prime}, w\right),
\end{aligned}
$$

where $C_{C P D}^{R}$ is the computation cost of the detection in the relay, i.e. a partial $K$-best detection with $K_{r}$. The $C_{C P D}^{D}$ represents the computation cost in the destination, which comprises of two consecutive $K$-best detection stages, each followed by an LLR calculation block, based on Eq. (10).

The LLR value computation count is given by

$$
C_{L L R}\left(M_{s}, K, w\right)=\left(\log _{2} w\right) \cdot M_{s} \cdot\left\{\gamma+2 \beta f_{1}\left(K w^{\prime}\right)\right\},
$$


where we use $\gamma$ and $\beta$ to represent the hardware-oriented costs for one real-valued multiplier and one real-valued compare-select operation, respectively.

Furthermore, for the full detect-and-forward (FDF), which is based on full symbol-level detection of all the antenna streams in the relay and a symbol-level maximal ratio combining in the destination followed by a K-best detection, the computation costs in the relay and destination are given by

$$
\begin{aligned}
C_{F D F}^{R}\left(M_{s}, w, \mathbf{k}_{F D F}\right) & =C_{K-\text { best }}\left(M_{s}, K_{r}^{\prime}, w\right), \\
C_{F D F}^{D}\left(M_{s}, w, \mathbf{k}_{F D F}\right) & =C_{K-\text { best }}\left(M_{s}, K_{d}^{\prime}, w\right) \\
& +C_{M R C}\left(M_{s}\right) \\
& +C_{L L R}\left(M_{s}, K_{d}^{\prime}, w\right),
\end{aligned}
$$

where the first terms on the RHS of both equations correspond to the detection in the relay and destination; respectively. The $K$ values for the full $K$-best detection steps in the relay and the destination are denoted by $\mathbf{k}_{F D F}=\left(K_{r}^{\prime}, K_{d}^{\prime}\right)$. The $C_{M R C}$ and $C_{L L R}$ represent the computational cost of computing the maximalratio combining (MRC) and LLR values. The $C_{M R C}$, which consists of two complex-valued matrix-by-vector multiplications and one complex-valued matrix addition, is given by

$C_{M R C}\left(M_{s}\right)=\theta\left\{2\left(2 M_{s}^{2}+2 M_{s}\left(M_{s}-1\right)+M_{s}\right)\right\}+\gamma\left\{2 \times 4 M_{s}^{2}\right\}$,

where $\theta$ and $\gamma$ represent the hardware-oriented costs for one realvalued adder and one real-valued multiplication operation, respectively.

The complexity of the relay, $C_{C P D}^{R}$, using the proposed CPD, for different values of expansion factor and modulation orders, is shown in Figure 4. A 4-antenna system of source, relay and destination is assumed. Note that the final point, for $e f=4$, corresponds to the full detect-and-forward computation count, $C_{F D F}^{R}$. From this figure, it is clear that performing a partial detection, as opposed to full detection, can save significant computational processing in the relay.

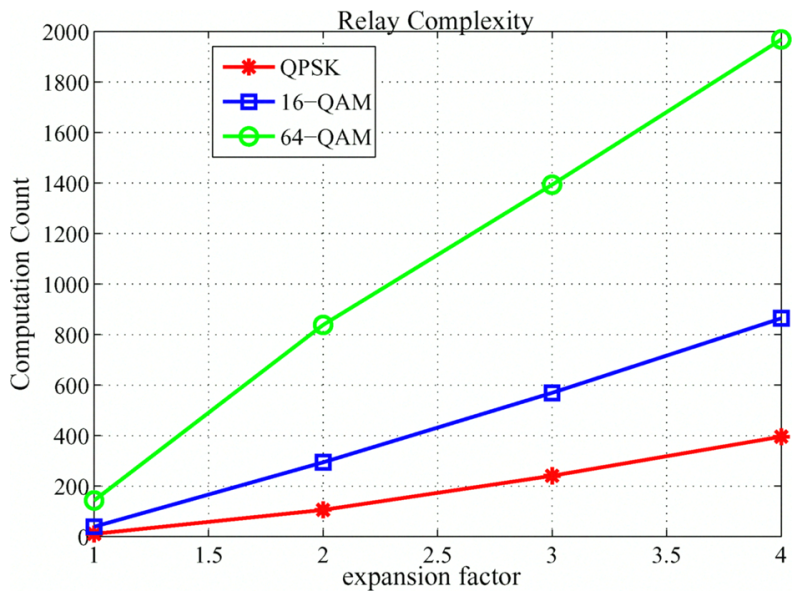

Fig. 4. The relay complexity vs. the expansion factor for $K_{r}=5$. The adder, compare-select and multiplier costs are assumed to be $\theta=\beta=1$ and $\gamma=10$.

\section{Simulation RESUlTS}

In this section, we present the simulation results for the proposed cooperative partial detection scheme. We show the BER performance improvement for different scenarios. Throughout this section, we set the detection parameters, namely $K$ values for the $K$-best and partial $K$-best detections, in such a way that the direct-link has similar computational complexity as the computation of the destination in the cooperation mode. In other words, using the results of section VI, we set $K_{d l}, \mathbf{k}_{F D F}=\left(K_{r}^{\prime}, K_{d}^{\prime}\right)$ and the $\mathbf{k}_{C P D}=\left(K_{r}, K_{d r}, K_{d s}\right)$ triple such that

$$
\begin{aligned}
C_{C P D}^{D}\left(M_{s}, w, \mathbf{k}_{C P D}\right) & =C_{F D F}^{D}\left(M_{s}, w, \mathbf{k}_{F D F}\right) \\
& =C_{K-\text { best }}\left(M_{s}, w, K_{d l}\right)
\end{aligned}
$$

where $K_{d l}$ is the $K$ value for the $K$-best detection in the direct-link scenario. Eq. (21) guarantees a fair comparison between the noncooperation case, the full detect-and-forward case, and the proposed cooperative partial detection scheme.

In terms of the network topology, we assume a three node relay network with the relay located between the source and destination, on the same line, and thus $d_{s d}=d_{s r}+d_{r d}$. We further assume that the path loss exponent is fixed to $\alpha=3$. The hardware-oriented complexity parameters for the real-valued adder, compare-select and multiplier are set to $\theta=\beta=1$ and $\gamma=10$. A rate $R=1 / 2$ turbo code [17] with memory length of 2 and the generating polynomials of $G_{1}(D)=1+D+D^{2}$ and $G_{2}(D)=1+D^{2}$ is used. The maximum number of inner iterations inside the turbo decoder is set to 8 , and the detector to decoder is a single phase iteration, i.e. no feedback from the decoder to the soft detector. The information message length for each frame is of size 9355 bits.

Figure 5 shows the performance improvement due to using the proposed cooperative partial detection scheme where the relay node is located in the first half of the line connecting the source and the destination, $d_{s r}=0.4$. In order to guarantee the equal computation constraint of Eq. $(21)$, we set $\mathbf{k}_{C P D}=(5,13,13)$ for the cooperative partial MIMO detection and $K_{d l}=10$ for the direct link. Two of the streams are detected in the relay, i.e. $e f=2$. Furthermore, in order to provide another comparison point, the BER of full detectand-forward of a relay in the same location with varying transmit power ratio, $\mu$, is presented as well. Note that for the full detect-andforward case, the $K$ values in the relay and the destination are set to $\mathbf{k}_{F D F}=(5,7)$ in order to guarantee that the full detect-and-forward scheme undertakes similar computation overhead in the destination.

Note that the proposed cooperative partial offers a $2.5 \mathrm{~dB}$ performance improvement over the non-relay, i.e. direct link, scenario, with limited complexity overhead. As shown in the previous section, the relay would have required three times higher complexity to achieve this performance. Figure 6 shows a similar behavior for a $5 \times 5$ system, and a relay detecting only ef $=3$ streams out of the 5 streams.

\section{CONCLUSION}

In this paper, a novel and practical cooperative partial detection scheme for MIMO relay networks was proposed; furthermore, the proposed scheme was based on architecture-friendly MIMO detection scenarios. The detection in the destination node was completed in two stages, and it was shown that with no extra computational complexity and transmit power, we can achieve a better BER performance compared to non-relay scenarios. Thus, this scheme can be adopted in resource-constrained MIMO relays to improve the link's performance without sacrificing significant relay resources.

\section{ACKNOWLEDGEMENT}

This work was supported in part by Nokia, and by NSF under grants EIA-0321266, CCF-0541363, CNS-0551692, and CNS0619767. 


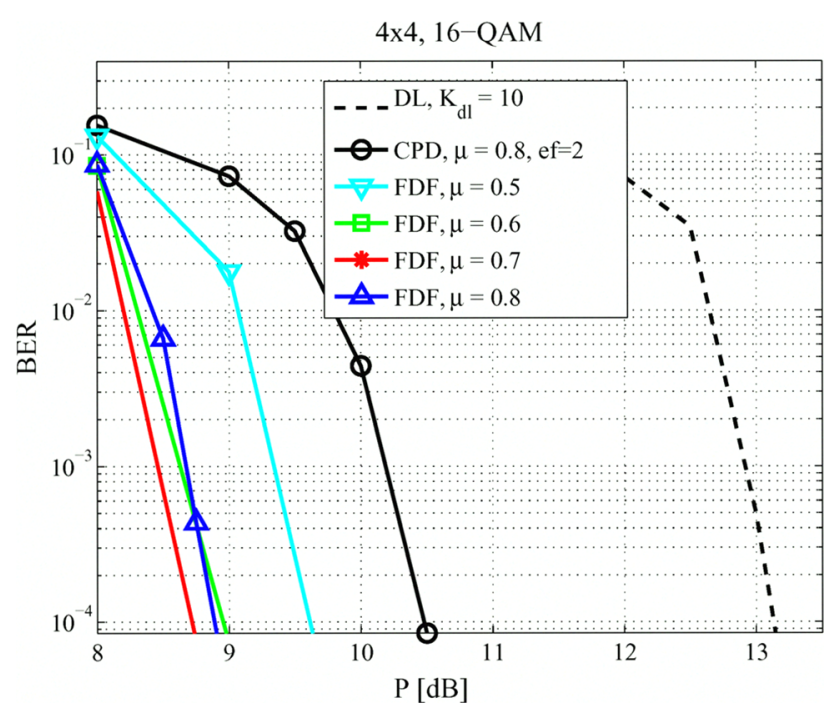

Fig. 5. BER of $4 \times 4,16$-QAM system with the relay located at $d_{s r}=0.4$ from the source, and for different power splitting ratios, $\mu=0.5, \ldots, 0.8$, between the source and the relay for the FDF case. Setting $\mathbf{k}_{C P D}=(5,13,13)$, $K_{d l}=10$ and $\mathbf{k}_{F D F}=(5,7)$ guarantees equal computational complexity for all the three scenarios in the destination. For the cooperative partial MIMO detection, the expansion factor is set to $e f=2$; therefore, the relay detects and forwards 2 of the streams to the destination, and the destination detects those two streams from the relay followed by the remaining two using the original vector it received from the source.

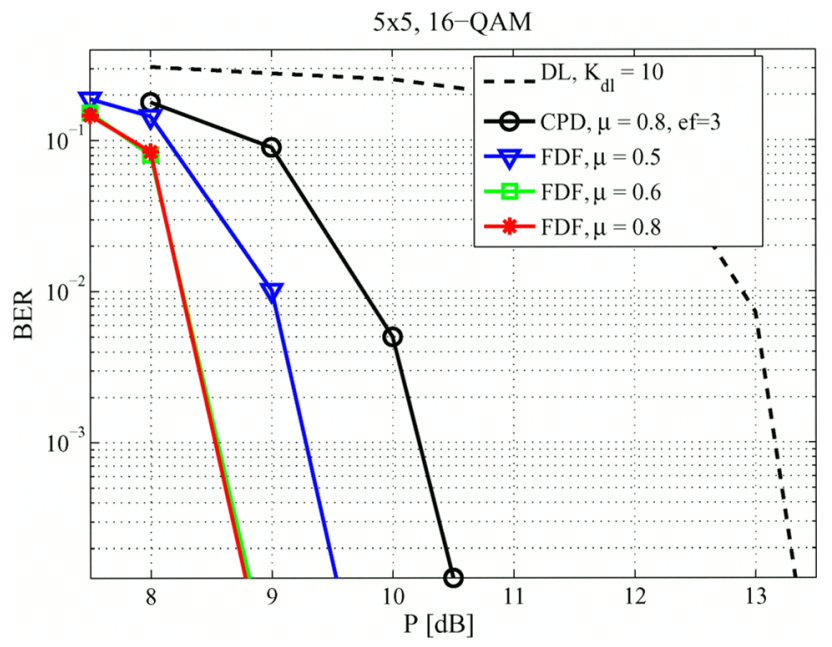

Fig. 6. BER of $5 \times 5,16$-QAM system with the relay located at $d_{s r}=0.4$ from the source, and for different power splitting ratios, $\mu=0.5, \ldots, 0.8$, between the source and the relay for the FDF case. Setting $\mathbf{k}_{C P D}=(5,13,13)$, $K_{d l}=10$ and $\mathbf{k}_{F D F}=(5,7)$ guarantees equal computational complexity for all the three scenarios in the destination. For the cooperative partial MIMO detection, the expansion factor is set to $e f=3$; therefore, the relay detects and forwards 2 of the streams to the destination, and the destination detects those two streams from the relay followed by the remaining two using the original vector it received from the source.

\section{REFERENCES}

[1] A. Sendonaris, E. Erkip and B. Aazhang, "User cooperation diversity part I: System Discription," IEEE Trans. on Communications, vol. 51, no. 11, pp. 1927-1938, Nov. 2003.

[2] J. N. Laneman, "Cooperative diversity in wireless networks: algorithms and architectures," Ph.D. dissertation, Massachusetts Institute of Technology, 2002.

[3] I. E. Telatar, "Capacity of multiantenna Gaussian channels," Europ. Trans. Telecommun., vol. 10, pp. 585-595, Nov. 1999.

[4] K. Wong, C. Tsui, R. S. Cheng and W. Mow, "A VLSI architecture of a K-best lattice decoding algorithm for MIMO channels," IEEE Int. Symp. Circuits Syst., vol. 3, pp. 273-276, May 2002.

[5] Z. Guo and P. Nilsson, "Algorithm and implementation of the K-Best sphere decoding for MIMO detection," IEEE JSAC, vol. 24, no. 3, pp. 491-503, Mar. 2006.

[6] K. Amiri and J. R. Cavallaro, "FPGA implementation of dynamic threshold sphere detection for MIMO systems," 40th Asilomar Conf on Signals, Systems and Computers, pp. 94-98, Nov 2006.

[7] B. Wang, J. Zhang and A. Host-Madsen, "On the capacity of MIMO relay channels," IEEE Trans. on Info Theory, vol. 51, no. 1, pp. 29-43, Jan. 2005.

[8] C. Lo, S. Vishwanath and R. W. Heath, "Rate bounds for mimo relay channels using precoding," IEEE GLOBECOM, 2005.

[9] H. Bolcskei, R. U. Nabar, O. Oyman and A. J. Paulraj, "Capacity scaling laws in MIMO relay networks," IEEE Trans. on Wireless Comm., vol. 5, no. 6, pp. 1433-1444, Jun. 2006.

[10] U. Fincke and M. Pohst, "Improved methods for calculating vectors of short length in a lattice, including a complexity analysis," Math. Computat., vol. 44, no. 170, pp. 463-471, Apr. 1985.

[11] M. Wenk, M. Zellweger, A. Burg, N. Felber and W. Fichtner, "K-best MIMO detection VLSI architecture achieving upto $424 \mathrm{Mbps,"} \mathrm{IEEE}$ ISCAS, pp. 1151-1154, May 2006.

[12] B. Hochwald and S. ten Brink, "Achieving near-capacity on a multipleantenna channel," IEEE Trans. on Comm., vol. 51, pp. 389-399, Mar. 2003.

[13] K. Amiri, C. Dick, R. Rao and J. R. Cavallaro, "Novel sort-free detector with modified real-valued decomposition (M-RVD) ordering in MIMO systems," Proc. of IEEE Globecom, Dec. 2008.

[14] S. S. Y. Dai and Z. Lei, "Performance comparison between coded VBLST and GSTTC MIMO-OFDM systems," 15th IEEE International Symp. on Personal Indoor and Mobile Radio Communications, pp. 25812585, Sep. 2004.

[15] — "A comparative study of QRD-M detection and sphere decoding for MIMO-OFDM systems," 16th IEEE International Symp. on Personal Indoor and Mobile Radio Communications, pp. 186-190, Sep. 2005.

[16] K. Amiri, C. Dick, R. Rao and J. R. Cavallaro, "A High Throughput Configurable SDR Detector for Multi-user MIMO Wireless Systems," Springer Journal of Signal Processing Systems, 2009.

[17] "Coded Modulation Library

http://www.iterativesolutions.com/Matlab.htm.” 